Mass Profiles and Shapes of Cosmological Structures

G. Mamon, F. Combes, C. Deffayet, B. Fort (eds)

EAS Publications Series, Vol. ?, 2005

\title{
UNDERSTANDING THE EQUILIBRIUM STRUCTURE OF CDM HALOS
}

\author{
Paul R. Shapiro ${ }^{1}$, Kyungjin Ahn ${ }^{1}$, Marcelo Alvarez ${ }^{1}$, Ilian T. Iliev ${ }^{2}$ and \\ Hugo Martel ${ }^{3}$
}

\begin{abstract}
N-body simulations find a universal structure for the halos which result from the nonlinear growth of Gaussian-random-noise density fluctuations in the CDM universe. This talk summarized our attempts to derive and explain this universal structure by analytical approximation and simplified models. As an example, we show here that a $1 \mathrm{D}$ spherical infall model involving a fluid approximation derived from the Boltzmann equation can explain not only the halo density profile but its phase-space density profile, as well.
\end{abstract}

\section{Introduction}

Numerical N-body simulations of structure formation from Gaussian-random-noise initial conditions in the CDM universe find a universal structure for halos. This universality is a fundamental prediction of the CDM model, but our knowledge is limited to the "empirical" N-body results, with little analytical understanding. In his talk, Shapiro summarized our attempts to fill this gap by a hierarchy of approximations, each simpler than the last: 1 . 3D gas/N-body simulations of halo formation from simplified initial conditions; 2. 1D, spherical, analytical models using a fluid dynamics approximation derived from the Boltzmann equation; 3. An analytical hydrostatic equilibrium model which follows from the virialization of top-hat density perturbations.

Most of the work described in that talk is summarized in Shapiro et al. (2004) and references therein and in Ahn \& Shapiro (2005), with the exception of our new results on the halo phase-space density profile, which we shall present here

\footnotetext{
${ }^{1}$ Department of Astronomy, 1 University Station, C1400, Austin, TX 78712

2 Canadian Institute for Theoretical Astrophysics, University of Toronto, 60 St. George Street, Toronto, ON M5S 3H8, Canada

${ }^{3}$ Département de physique, de génie physique et d'optique, Université Laval, Québec, QC G1K 7P4, Canada
} 
Mass Profiles and Shapes of Cosmological Structures

for the first time. Due to length limitations, we shall limit this paper to just a few items from category 2 above. A more complete version of Shapiro's talk is available at the meeting website ${ }^{1}$.

\section{Universal Structure of CDM Halos: N-body Results}

CDM N-body halos show universal mass profiles. The same density profile fits halos from dwarf galaxies to clusters, independent of halo mass, of the shape of the density fluctuation power spectrum $P(k)$, and of background cosmology: $\rho(r) / \rho_{-2}=f c n\left(r / r_{-2}\right)$, where $r_{-2} \equiv$ radius where $d \ln \rho / d \ln r=-2$ and $\rho_{-2} \equiv$ $\rho\left(r_{-2}\right)$ (e.g. Navarro et al. 2004) $)^{2}$. As $r \rightarrow \infty, \rho \rightarrow r^{-3}$, while as $r \rightarrow 0, \rho \rightarrow r^{-\alpha}$, $1 \leq \alpha \leq 1.5$ (e.g. Navarro, Frenk, \& White 1997; Moore et al. 1999). Diemand, Moore \& Stadel (2004) report that

$$
\rho_{\alpha \beta \gamma}=\frac{\rho_{s}}{\left[r / r_{s}\right]^{\gamma}\left[1+\left(r / r_{s}\right)^{\alpha}\right]^{(\beta-\gamma) / \alpha}}
$$

with $(\alpha, \beta, \gamma)=(1,3, \gamma)$ summarizes the fits to current simulations, with $\gamma_{\text {best-fit }}=$ $1.16 \pm 0.14$.

The profiles of individual halos evolve with time. The halo mass grows as $M(a)=M_{\infty} \exp \left[-S a_{f} / a\right]$, where $S \equiv[d \ln M / d \ln a]\left(a=a_{f}\right)=2$ (Wechsler et al. 2002). The density profile concentration parameter, $c=r_{200} / r_{s}$, also grows, according to $c(a)=c\left(a_{f}\right)\left(a / a_{f}\right)$ for $a>a_{f}$ (Bullock et al. 2001; Wechsler et al. 2002), starting from $c(a) \leq 3-4$ for $a \leq a_{f}$ (initial phase of most rapid mass assembly) (Tasitsiomi et al. 2004).

CDM N-body halos show surprisingly isotropic velocity distributions. Halos have universal velocity anisotropy profiles, $\beta\left(r / r_{200}\right)$, where $\beta(r) \equiv 1-\left\langle v_{t}^{2}\right\rangle /\left(2\left\langle v_{r}^{2}\right\rangle\right)$, with $\beta=0$ (isotropic) at $r=0$, gradually rising to $\beta \sim 0.3$ at $r=r_{200}$ (e.g. Carlberg et al. 1997).

CDM N-body halos show universal phase-space density profiles. N-body results find $\rho / \sigma_{\mathrm{V}}^{3} \propto r^{-\alpha_{\mathrm{ps}}}$, where $\alpha_{\mathrm{ps}}=1.875$ (Taylor \& Navarro 2001), $\alpha_{\mathrm{ps}}=1.95$ (Rasia, Tormen and Moscardini 2004), and $\alpha_{\mathrm{ps}}=1.9 \pm 0.05$ (Ascasibar et al. 2004). (Also related: $P(f) \propto f^{-2.5 \pm 0.05}$; Arad, Dekel, \& Klypin 2004).

\section{The Fluid Approximation: 1D Halo Formation From Cosmological Infall}

The collisionless Boltzmann equation and the Poisson equation can be used to derive exact dynamical equations for CDM which are identical to the fluid conservation equations for an ideal gas with adiabatic index $\gamma=5 / 3$, if we assume spherical

\footnotetext{
${ }^{1}$ http://www2.iap.fr/users/gam/yappa-ng/index.php?album=\%2FIAP05\%2F\&image=Shapiro.pdf

${ }^{2}$ The weak mass-dependence suggested by Ricotti (2003) is an exception which remains to be confirmed.
} 

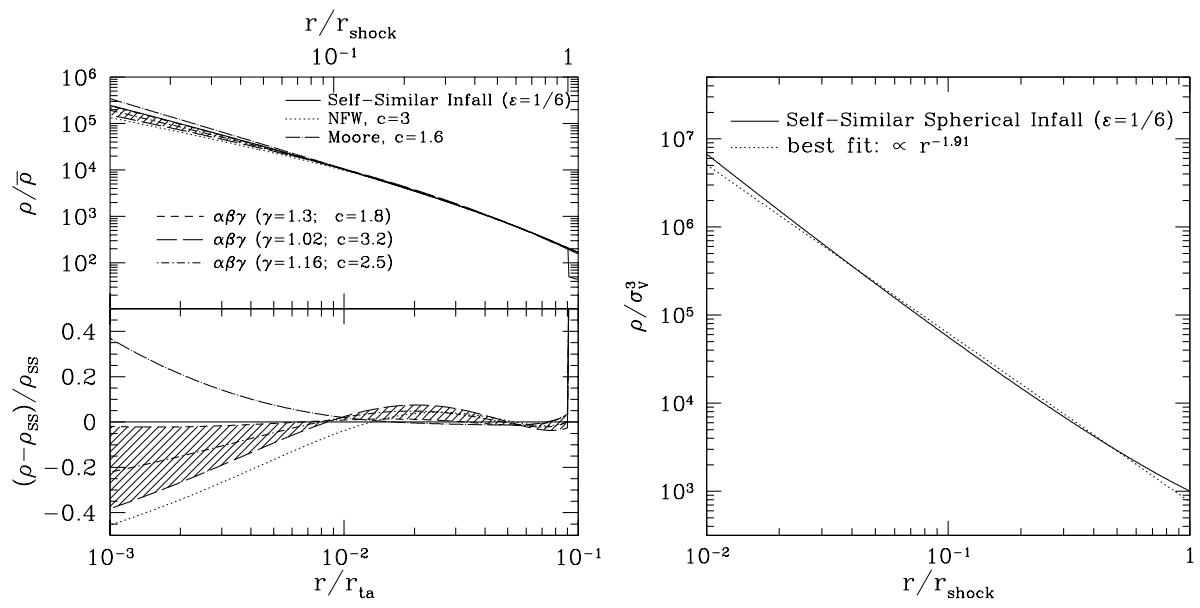

Fig. 1. Self-similar Spherical Infall with $\varepsilon=1 / 6$. (a) (left) (top) Halo mass density versus radius for analytical, similarity solution in the fluid approximation, compared with best-fitting NFW, Moore and $\alpha \beta \gamma$ profiles with $(\alpha, \beta, \gamma)=(1,3, \gamma)$. (bottom) fractional deviation of fits from self-similar solution $\rho_{\mathrm{SS}}$. (b) (right) Halo phase-space density versus radius for analytical similarity solution compared with best fitting power-law $r^{-1.91}$.

symmetry and a velocity distribution which is both skewless and isotropic, assumptions which approximate the N-body results reasonably well (Ahn \& Shapiro 2005). We have used this fluid approximation to show that most of the universal properties of CDM N-body halos described above can be understood as the dynamical outcome of continuous cosmological infall.

\subsection{Self-similar gravitational collapse: the spherical infall model}

In an EdS universe, scale-free, spherically symmetric perturbations $\delta M / M \propto M^{-\epsilon}$ result in self-similar structure formation. Each spherical mass shell around the center expands until it reaches a maximum radius $r_{\mathrm{ta}}$ and recollapses, $r_{\mathrm{ta}} \propto t^{\xi}$, $\xi=(6 \epsilon+2) /(9 \epsilon)$. There are no characteristic length or time scales besides $r_{\mathrm{ta}}$ and Hubble time $t$. For cold, unperturbed matter, this results in highly supersonic infall, terminated by a strong, accretion shock which thermalizes the kinetic energy of collapse: $r_{\text {shock }}(t) \propto r_{\mathrm{ta}}(t)$. The spherical region bounded by the shock is roughly in hydrostatic equilibrium, a good model for virialized halos.

Consider halo formation around peaks of the Gaussian random noise primordial density fluctuations. If $P(k) \propto k^{n}$, then $\nu \sigma$-peaks with $\nu \geq 3$ have simple powerlaw profiles for accumulated overdensity inside $r, \Delta_{0}(r)=\delta M / M \propto r^{-(n+3)} \propto$ $M^{-(n+3) / 3}$ (e.g. Hoffman \& Shaham 1985), which implies self-similar infall with $\epsilon=(n+3) / 3$. For $\Lambda$ CDM, galactic halos are well approximated by $n=-2.5 \pm 0.2$ for $10^{3} \leq M / M_{\odot} \leq 10^{11}$. By applying the fluid approximation to the problem of self-similar spherical infall with $\epsilon=1 / 6$ (i.e. $n_{\mathrm{eff}}=-2.5$ ), Ahn \& Shapiro 

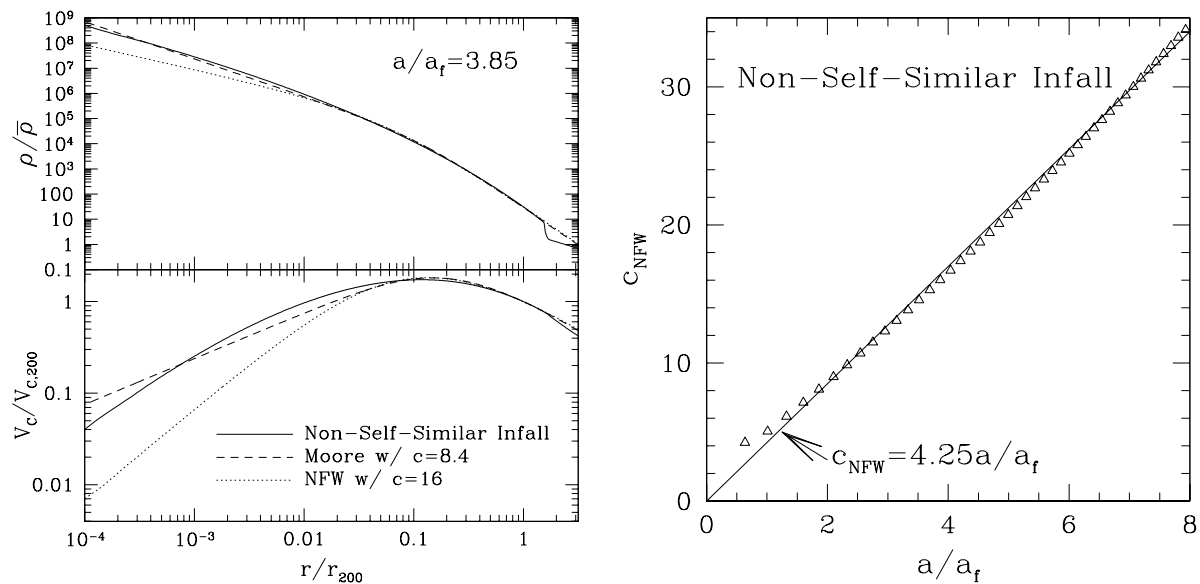

Fig. 2. Non-Self-Similar Spherical Infall. (a) (left) (top) Halo mass density versus radius at epoch $a / a_{\mathrm{f}}=3.85$, according to fluid approximation solution for the non-self-similar spherical infall rate which makes halo mass grow in time like Wechsler et al. (2002) fitting formula, compared with best-fitting NFW and Moore profiles; (bottom) Corresponding halo rotation curves. (b) (right) Concentration parameter versus scale factor for the bestfitting NFW profiles at each time-slice during the evolution of the fluid approximation solution for time-varying spherical infall at the Wechsler et al. (2002) rate.

(2005) derived a 1D, analytical solution for halo formation and evolution, in which $r_{\text {shock }} \propto r_{\mathrm{ta}} \propto t^{2}$, and $M \propto t^{4}$. The resulting self-similar halo density profile inside the accretion shock agrees with that of CDM N-body halos, with a bestfit $\alpha \beta \gamma$-profile which has $(\alpha, \beta, \gamma)=(1,3,1.3)$ (see Figure $1(\mathrm{a})$ ). As we show in Figure 1(b), this analytical similarity solution for $\epsilon=1 / 6$ also derives the universal phase-space density profile found for CDM N-body halos, $\rho / \sigma_{\mathrm{V}}^{3} \propto r^{-\alpha_{\mathrm{ps}}}, \alpha_{\mathrm{ps}} \simeq 1.9$.

\subsection{Non-self-similar infall: Mass Assembly History and the Evolution of CDM $\mathrm{N}$-body Halo Profiles}

Self-similar infall may provide a good explanation for some halo properties, but it cannot explain how profile shapes change with time and depart from self-similarity. To do this, we have derived the perturbation profile that leads to the non-selfsimilar halo mass growth rate reported for CDM N-body halos by Wechsler et al. (2002) and used the fluid approximation to derive the halo properties that result (Alvarez, Ahn, \& Shapiro 2003). We solved the fluid approximation equations by a 1D, spherical, Lagrangian hydro code. These solutions explain most of the empirical CDM N-body halo properties and their evolution described above as a dynamical consequence of this time-varying, but smooth and continuous infall rate. The halo density profiles which result are well fit by (and intermediate between) NFW and Moore profiles (over the range $r / r_{200} \geq 0.01$ ) (see Figure $2(\mathrm{a})$ ). 


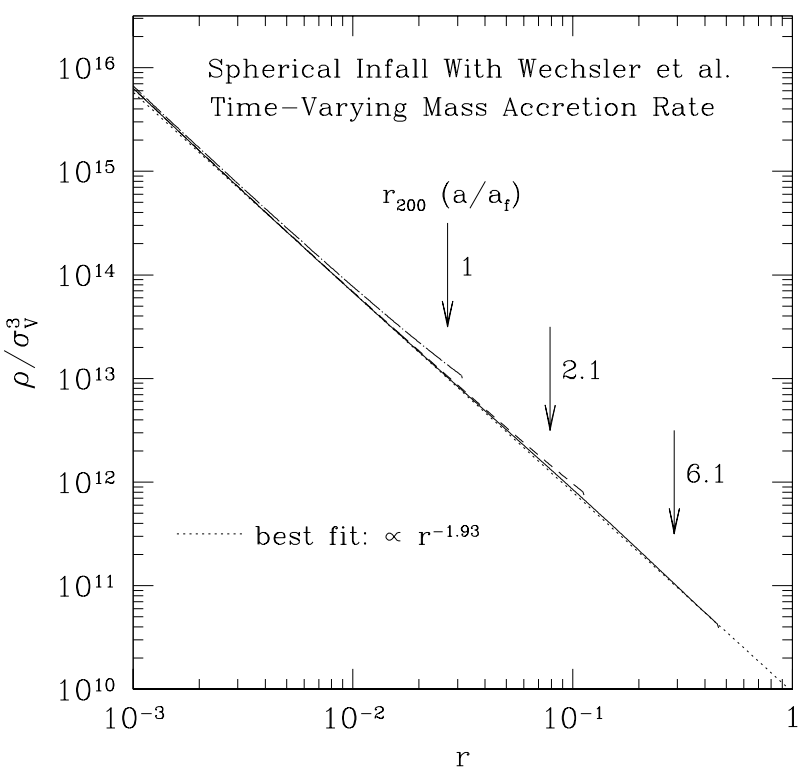

Fig. 3. Phase-space density profiles for the same non-self-similar infall solution plotted in Figure 2, for $a / a_{\mathrm{f}}=1,2.1$, and 6.1, with arrows showing locations of $r_{200}$ at each epoch, along with best-fitting power-law $r^{-1.93}$.

These halo density profiles evolve just like CDM N-body halos, too. The halo concentration parameter grows with time just like CDM N-body halos (see Figure 2(b)). In addition, these solutions yield a halo phase-space density profile, $\rho / \sigma_{v}^{3}$, in remarkable agreement at all times with the universal profile reported for CDM N-body halos (see Figure 3). We therefore conclude that the time-varying mass accretion rate, or equivalently the shape of the initial density perturbation, is the dominant influence on the structure of CDM halos, which can be understood simply in the context of spherical collapse and the accretion of a smoothly-distributed, spherically-symmetric collisionless fluid.

This work was supported NASA Astrophysical Theory Program grants NAG510825, NAG5-10826, NNG04GI77G, Texas Advanced Research Program grant 3658-0624-1999, and a Department of Energy Computational Science Graduate Fellowship to M.A.A.

\section{References}

Ahn, K., \& Shapiro, P.R., 2005, MNRAS, in press, astro-ph/0412169

Alvarez, M. A., Ahn, K., \& Shapiro P. R., 2003, RevMexAA, 18, 4 
Ascasibar, Y., Yepes, G., Gottlöber, S., \& Müller, V., 2004, MNRAS, 352, 1109

Arad, I., Dekel, A., \& Klypin, A., 2004, MNRAS, 353, 15

Bullock, J. S., Kolatt, T. S., Sigad, Y., Somerville, R. S., Kravtsov, A. V., Klypin, A. A., Primack, J. R., \& Dekel, A., 2001, MNRAS, 321, 559

Carlberg, R. G., Yee, H. K. C., Ellingson, E., Morris, S. L., Abraham, R., Gravel, Pl, Pritchet, C. J., Smecker-Hane, T., Hartwick, F. D. A., Hesser, J. E., Hutchings, J. B., \& Oke, J. B., 1997, ApJ, 485, L13

Diemand, J., Moore, B., \& Stadel, J., 2004, MNRAS, 353, 624

Hoffman, Y. \& Shaham, J. 1985, ApJ, 297, 16

Navarro, J. F., Frenk, C. S., \& White, S. D. M. 1997, ApJ, 490, 493

Navarro, J. F., Hayashi, E., Power, C., Jenkins, A. R., Frenk, C. S., White, S. D. M., Springel, V., Stadel, J., \& Quinn, T. R., 2004, MNRAS, 349, 1039

Moore, B., Ghigna, S., Governato, F., Lake, G., Quinn, T., Stadel, J., \& Tozzi, P., 1999, ApJ, 524, L19

Rasia, E., Tormen, G., \& Moscardini, L., 2004, MNRAS, 351, 237

Ricotti, M., 2003, MNRAS, 344, 1237

Shapiro, P.R., Iliev, I., Martel, H., Ahn, K., and Alvarez, M., 2004, in Progress in Dark Matter Research (Nova Science Publishers, Inc.), in press (astro-ph/0409173)

Tasitsiomi, A., Kravtsov, A. V., Gottlöber, S., \& Klypin, A. A. 2004, ApJ, 607, 125

Taylor, J. E., \& Navarro, J. F., 2001, ApJ, 563, 483

Wechsler, R. H., Bullock, J. S., Primack, J. R., Kravtsov, A. V., \& Dekel, A. 2002, ApJ, 568, 52 